Terr. Atmos. Ocean. Sci., Vol. 18, No. 3, 547-565, August 2007

\title{
Local Effects on Strain Seismogram at Matsushiro Seismological Observatory - 1. Love Waves
}

\author{
Taishi Okamoto ${ }^{1,}{ }^{*}$, Yutaka Ikegami ${ }^{1}$, and Kazuya Kokubo ${ }^{2,3}$
}

(Manuscript received 28 March 2006, in final form 13 November 2006)

\begin{abstract}
We proposed a simple method to evaluate local effects on a strain seismogram, and applied the method to investigation of the effects at Matsushiro Seismological Observatory, Japan Meteorological Agency, central Japan, for a Love wave in a period range of 170 - 400 sec. First, we took a ratio of $\mathrm{EW}$ to NS component strain for a Love wave on a timefrequency plane. Although NS and EW component strain for a Love wave travelling in any direction have the same amplitude theoretically, the ratio of observed EW to NS component strain was 0.7 . Next, we compared the strain seismogram with a velocity seismogram recorded with STS-1 broadband seismometer. A ratio of a partial derivative of a displacement field with respect to time to that with respect to wave propagation direction equals a phase velocity of the wave theoretically. Utilizing the fact, we estimated the phase velocity of a Love wave using the observed velocity and strain seismogram, after NS component strain had been multiplied by 0.7 . The result was $24 \%$ smaller than the theoretical phase velocity. Finally, we compared the velocity seismogram with other velocity seismograms recorded by STS-1 seismometers at F-net observation stations near Matsushiro and by STS-2 seismometer at Matsushiro, and found that the every deviation from Matsushiro was less than $10 \%$. From these results, we conclude that the EW and NS component strain seismograms have been amplified by factors of $\mathbf{1 . 3 2}$ and $\mathbf{1 . 8 8}$ for a Love wave, respectively. This fact, which may
\end{abstract}

${ }^{1}$ Department of Geophysics, Graduate School of Science, Kyoto University, Kyoto, Japan

2 Matsushiro Seismological Observatory, Japan Meteorological Agency, Nagano, Japan

${ }^{3}$ Seismological and Volcanological Department, Japan Meteorological Agency, Tokyo, Japan

* Corresponding author address: Prof. Taishi Okamoto, Department of Geophysics, Graduate School of Science, Kyoto University, Kyoto, Japan; E-mail: dollar@kugi.kyoto-u.ac.jp doi: 10.3319/TAO.2007.18.3.547(T) 


\begin{abstract}
be because of local geology and/or topography effects, must be taken into account when the strain seismogram is used for seismological applications such as the CMT inversion and research on earth's free oscillations.
\end{abstract}

(Key words: Strain seismogram, Love wave, Phase velocity)

\title{
1. INTRODUCTION
}

An extensometer, having a good response up to DC component, can be used as a very broadband seismometer if output signals are digitized at a high sampling rate. Short-period body waves, long-period surface waves, near-field terms, and Earth's free oscillations - all the seismic waves can be recorded by the instrument alone.

At Matsushiro Seismological Observatory, JMA, continuous observation of crustal movement has been performed by 100-meter-long 2-component quartz-tube extensometers (Yamagishi et al. 1976). These extensometers have been able to be treated as a wide dynamic range and very broadband seismograph since renewal of a telemeter system in 2001, which made ADC sampling rate and resolution 1-sec and 24-bit, respectively (Wakui et al. 2002). Ikegami (2006) analysed records observed by this strain seismograph from 2004 SumatraAndaman earthquake and found an abnormal strain in the record.

However, it has recently been reported that an observed strain seismogram is considerably affected by local geology structure. For example, Asai et al. (2005) found that co-seismic strain-steps associated with the 2004 off the Kii peninsula earthquakes observed at the same site but at different depths increase with depth. Okubo et al. (2005) also pointed out that shortperiod body waves from earthquakes observed by borehole strainmeters are affected by local media.

Thus it is very important to estimate the effects of local media on an observed strain seismogram before using the record for seismological applications. In the present study, we investigate the local effects on observed strain seismograms for long-period Love waves in a period range of 170 - $400 \mathrm{sec}$. Our goal is to obtain a correction factor which allows us to remove local effects from an observed strain seismogram.

\section{AMPLITUDE RATIO OF EW TO NS COMPONENT FOR A LOVE WAVE}

First, we investigate amplitude ratio of EW to NS component strain for a long-period Love wave. A Love wave involves no volume change and has no vertical displacement (and of course no vertical linear strain). In a Cartesian coordinate system where $z$-axis is taken vertically and $x$ - and $y$-axis perpendicular to each other within a horizontal plane, these are respectively expressed by $\varepsilon_{x x}+\varepsilon_{y y}+\varepsilon_{z z}=0$ and $\varepsilon_{z z}=0$, where $\varepsilon_{x x}$, $\varepsilon_{y y}$, and $\varepsilon_{z z}$ denote linear strains in $x$-, $y$-, and $z$-direction. From the two equations, we have $\varepsilon_{x x}=-\varepsilon_{y y}$, which means that two seismograms from 2-component extensometers perpendicular to each other have the same amplitude for a Love wave travelling in any direction (This fact has already been reported by Benioff and Gutenberg 1952). Note that the above relation is independent of not only wave propagation direction but also frequency. Hence if only Love waves can be extracted from EW 
and NS component strain seismograms, their amplitude ratio must be unity whether in the time domain or in the frequency domain.

On 23 December 2004, a very large earthquake with a moment magnitude of 8.1 occurred beneath north of Macquarie Island, New Zealand. This earthquake provided perfect conditions for the present analysis because its focal mechanism and source-receiver azimuth, shown in Fig. 1, made the amplitude of Love waves observed at Matsushiro very large and Rayleigh waves nearly zero due to their radiation patterns.

Before taking the ratio of the two-components observed strain seismograms from this earthquake, we first perform the same analysis for theoretical strain seismograms to verify the applicability of this method. The theoretical strain seismograms at Matsushiro, shown in Fig. 2, were synthesized based upon the normal mode theory (Gilbert and Dziewonski 1975), by superposition of eigenfunctions with periods longer than $45 \mathrm{sec}$. The Earth model PREM (Dziewonski and Anderson 1981) and the Harvard CMT solution are adopted in the calculation. The source parameters are summarized in Table 1, event (a). The theoretical strain seismograms are filtered in the period range of 171 - $400 \mathrm{sec}$ for a particle motion analysis and shown in Fig. 3, along with theoretical velocity seismograms and their particle motion diagrams. The particle motions for the velocity seismograms indicate that the seismograms are dominated by Love waves. It is also to be noted that EW component strain has the same amplitude as NS for the Love waves.

Figures $4 \mathrm{a}$ - $\mathrm{c}$ show spectrograms for the EW and NS component theoretical strain seismograms produced by multiple filter technique (Dziewonski et al. 1969), and their amplitude ratio, respectively. Theoretical group velocity dispersion curves for fundamental and first higher mode Love waves and fundamental mode Rayleigh waves are also plotted. Figures $4 \mathrm{a}$ and $\mathrm{b}$ again show that fundamental mode Love waves dominate the seismograms. Even first higher mode Love waves can be found in the both component spectrograms, while Rayleigh waves fail to appear except for a tiny amplitude R2 phase.

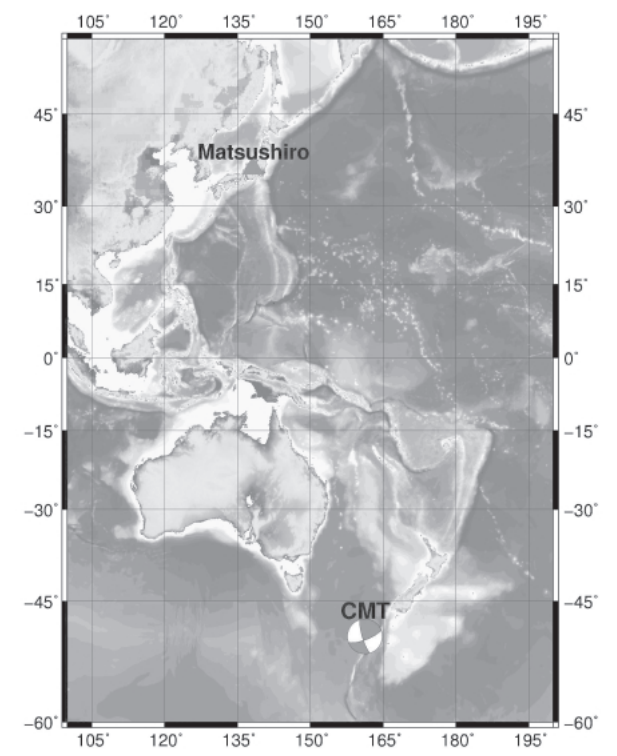

Fig. 1. Map of observation station and source of the 2004 North of Macquarie Island Earthquake. 
(a)

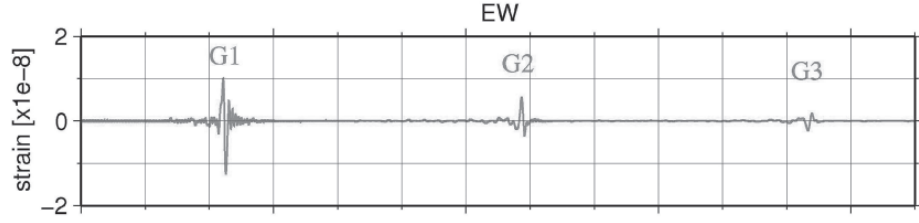

(b)

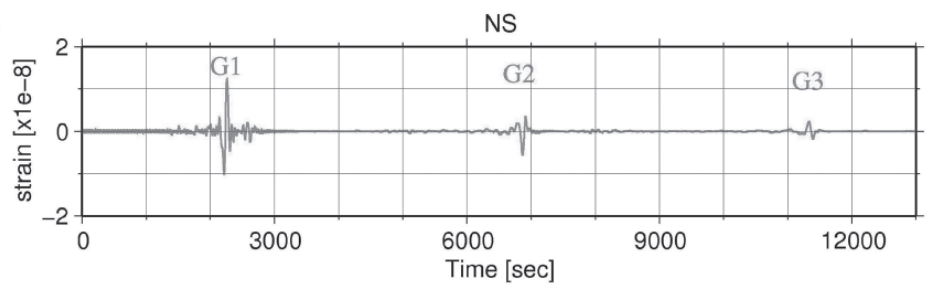

Fig. 2. Theoretical strain seismograms at Matsushiro for 2004 North of Macquarie Island earthquake. (a) EW and (b) NS component. The time is measured from 145931 UT 23 December 2004, corresponding to the centroid time.

Table 1. Harvard centroid moment tensor solutions for three events used in this study. (a) 2004 Macquarie Island Earthquake, (b) 2005 Northern Chile Earthquake, and (c) 2001 Southern Peru Earthquake.

\begin{tabular}{lrrr}
\hline Event & (a) & (b) & (c) \\
\hline Date & Dec. 23,2004 & June 13,2005 & June 23,2001 \\
Centroid Time & $14: 59: 30.9 \mathrm{UTC}$ & $22: 44: 40.2 \mathrm{UTC}$ & $20: 34: 23.3 \mathrm{UTC}$ \\
\hline Latitude & $49.91 \mathrm{~S}$ & $20.02 \mathrm{~S}$ & $17.28 \mathrm{~S}$ \\
Longitude & $161.25 \mathrm{E}$ & $69.23 \mathrm{~W}$ & $72.71 \mathrm{~W}$ \\
Depth & $27.5 \mathrm{~km}$ & $94.5 \mathrm{~km}$ & $29.6 \mathrm{~km}$ \\
\hline Mw & 8.1 & 7.8 & 8.4 \\
Half Duration & $26.7 \mathrm{sec}$ & $18.3 \mathrm{sec}$ & $43.2 \mathrm{sec}$ \\
\hline Moment Tensor & $\left(\times 10^{28}\right.$ dyne cm & $\left(\times 10^{27}\right.$ dyne cm $)$ & $\left(\times 10^{28} \mathrm{dyne} \mathrm{cm}\right)$ \\
$M_{r r}$ & 0.108 & -3.700 & 2.245 \\
$M_{\theta \theta}$ & 0.874 & -0.272 & -0.547 \\
$M_{\phi \phi}$ & -0.982 & 3.970 & -1.698 \\
$M_{r \theta}$ & 0.488 & 0.615 & 1.339 \\
$M_{r \phi}$ & -0.317 & -3.630 & -3.728 \\
$M_{\theta \phi}$ & & & 1.444 \\
\hline
\end{tabular}


(a)

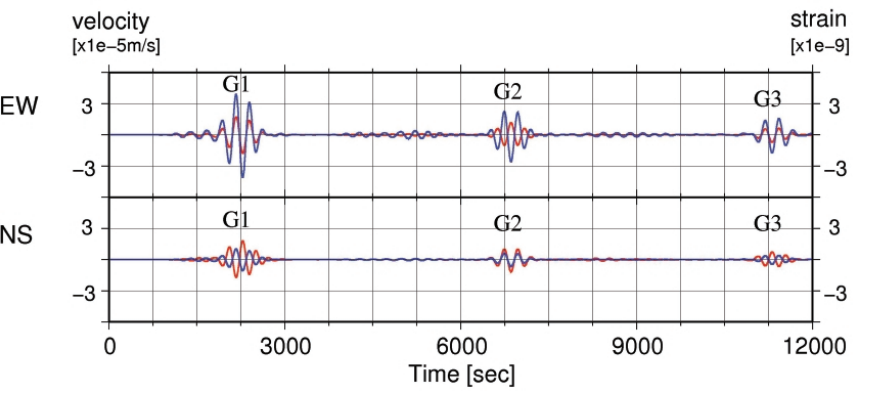

(b)

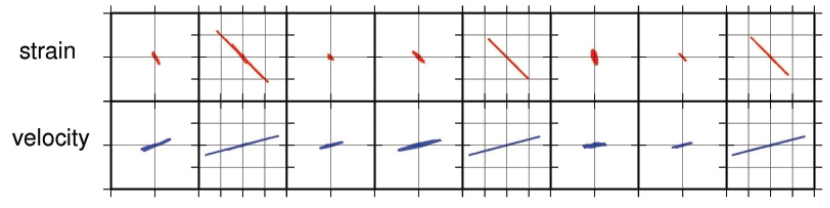

Fig. 3. Theoretical strain (red) and velocity (blue) seismograms filtered in a period range of 171 - $400 \mathrm{sec}$. (b) Particle motions for the strain (red) and velocity (blue) seismograms.
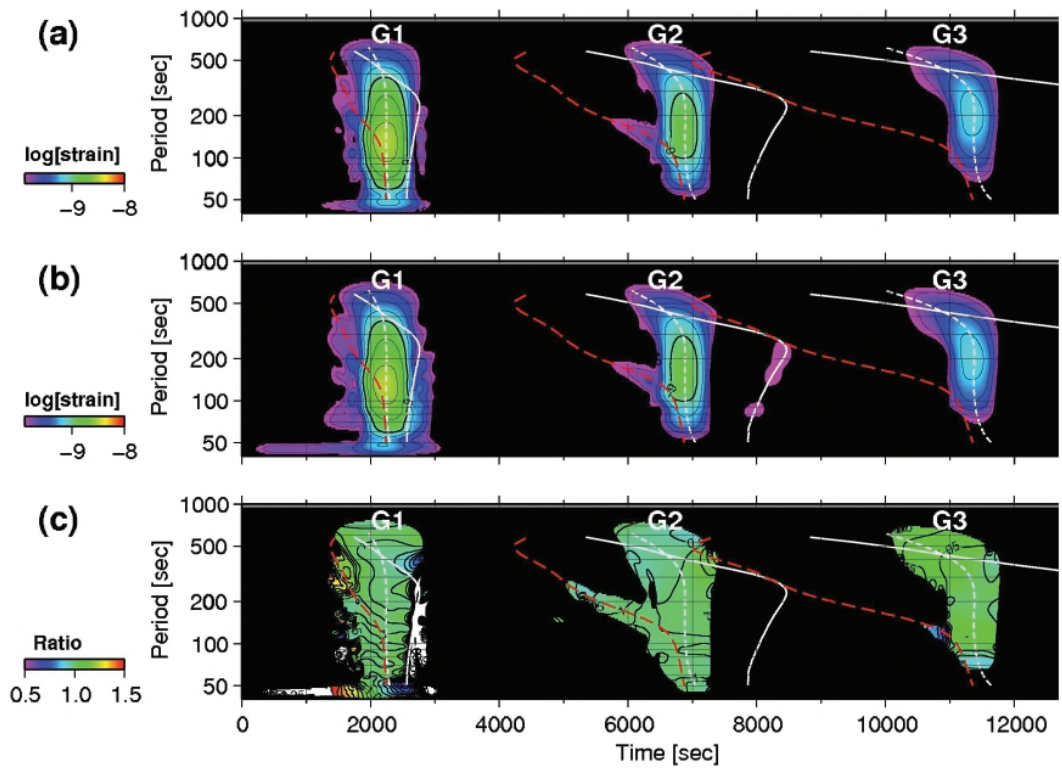

Fig. 4. Spectrograms for (a) EW and (b) NS component theoretical strain seismograms shown in Fig. 2, and (c) amplitude ratio of EW to NS component. The time is the same as Fig. 2. White dotted curves, red dotted curves, and white solid curves denote theoretical group velocity dispersion curves for multi-orbit fundamental mode Love waves (G1, G2, and G3), first higher mode Love waves, and fundamental mode Rayleigh waves in PREM. 
Figure 5 plots cross-sections of Fig. 4c along the positions of theoretical dispersion curves for G1, G2, and G3 phases. Note that ratio of EW to NS component for the theoretical strain seismogram is approximately unity as we expected, in the period range of $100-500 \mathrm{sec}$. The small departures from unity would be due to contamination from other phases; a fundamental mode Love wave has the same group velocity as a first higher mode Love wave in a period range of $50-100 \mathrm{sec}$ and as a fundamental mode Rayleigh wave around a period of $420 \mathrm{sec}$, as shown in Fig. 4. In addition, it is also known that in some cases the multiple filter technique cannot determine an amplitude of a mode of interest accurately (Herrmann 1973). The defect inherent in the technique also might have caused the small discrepancy.

Observed strain seismograms, shown in Fig. 6, are analysed in the same way. Figures 7a-c show spectrograms for the EW and NS component observed strain seismograms and their amplitude ratio, respectively. And Fig. 8 plots cross-sections of Fig. 7c along the positions of local maximum amplitudes corresponding to G1, G2, and G3 phases. It should be noted that ratio of EW to NS component for the observed strain seismogram is 0.7 in the period range of $100-500 \mathrm{sec}$, unlike the result for the theoretical seismogram. It seems that the ratio does not depend on the period. The observed strain seismograms are filtered in the period range of $171-400 \mathrm{sec}$ for a particle motion analysis and shown in Fig. 9, along with observed velocity seismograms and their particle motion diagrams. The particle motion analysis further confirms that the observed seismograms are dominated by Love waves and that the amplitude of NS component strain is significantly larger than that of EW for the Love waves.

The above analysis is also performed for two other events: Northern Chile Earthquake of 13 June 2005 and Southern Peru Earthquake of 23 June 2001. Source parameters of the two events are summarized in Table 1, events (b) and (c). Figures 10 and 11 show ratios of EW to NS component observed strain seismograms from 2005 Northern Chile Earthquake and from 2001 Southern Peru Earthquake, respectively. Both the figures illustrate that the ratios are

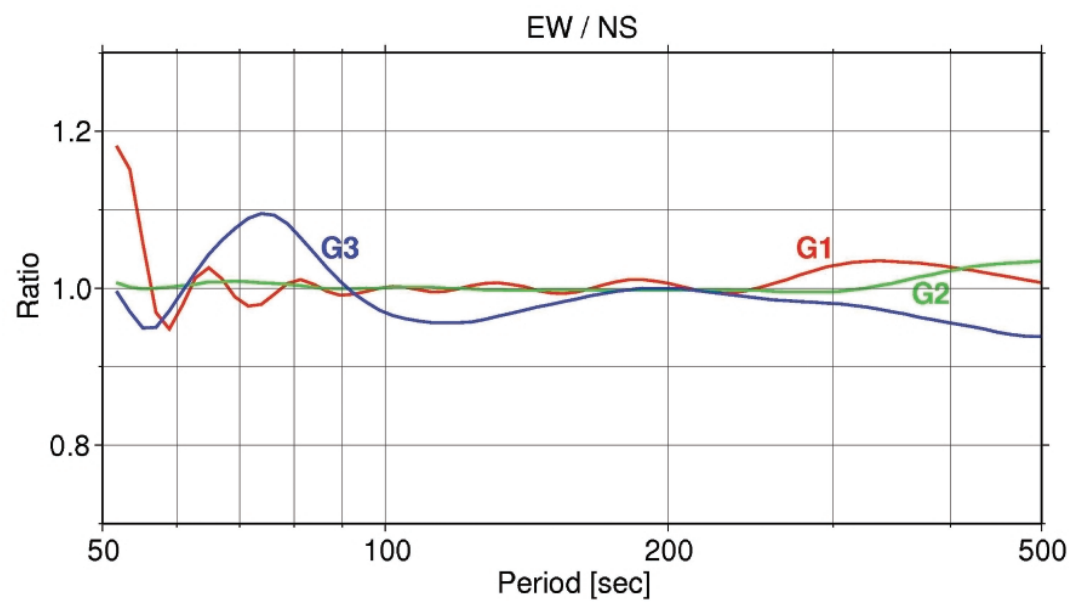

Fig. 5. Cross-sections of spectrograms in Fig. 4c calculated along the positions of theoretical dispersion curves for G1(red), G2(green), and G3(blue) phases. 
(a)

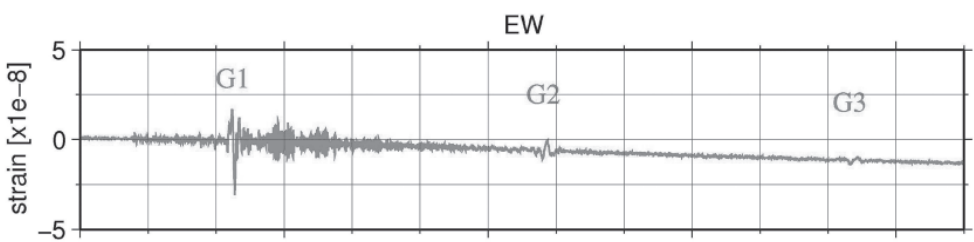

(b)

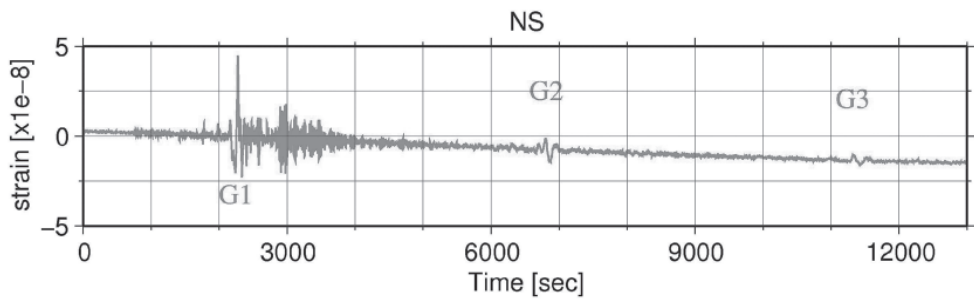

Fig. 6. Observed strain seismograms at Matsushiro from the 2004 North of Macquarie Island earthquake. (a) EW and (b) NS component. The time is the same as Fig. 2.
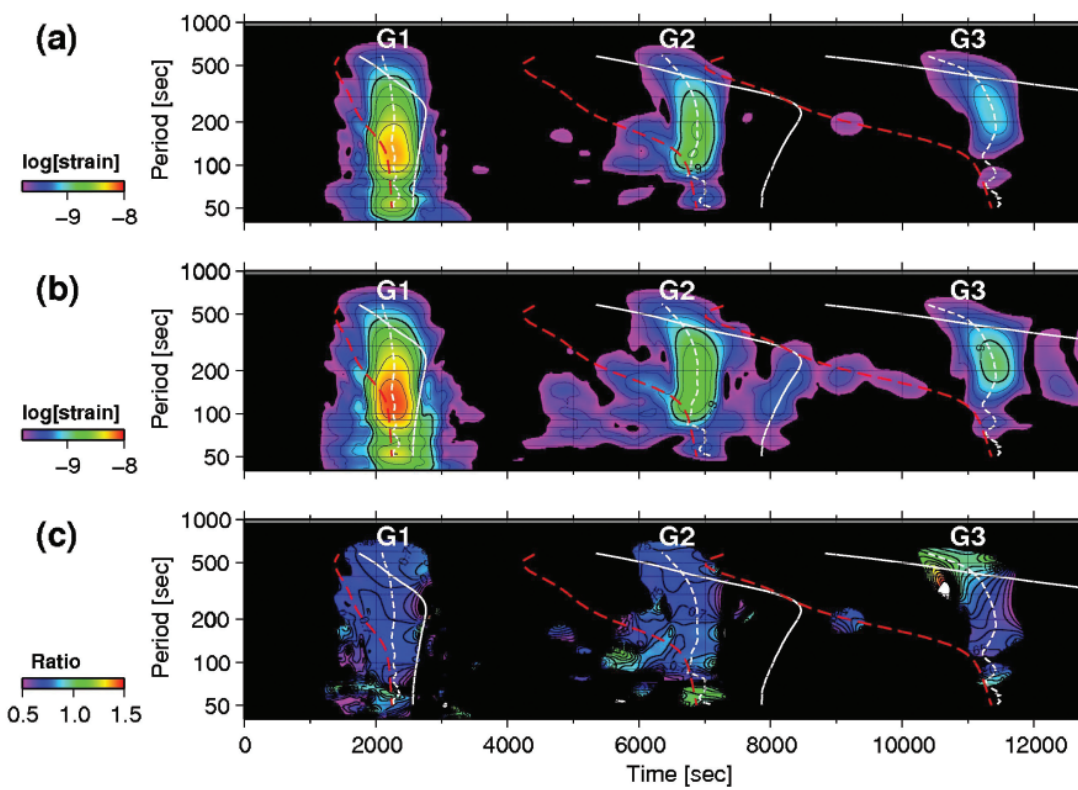

Fig. 7. Spectrograms for (a) EW and (b) NS component observed strain seismograms shown in Fig. 6, and (c) amplitude ratio of EW to NS component. The time and curves except for the white dotted curves are the same as in Fig. 4. The white dotted curves denote the position of local maximum amplitudes corresponding to G1, G2, and G3 phases. 


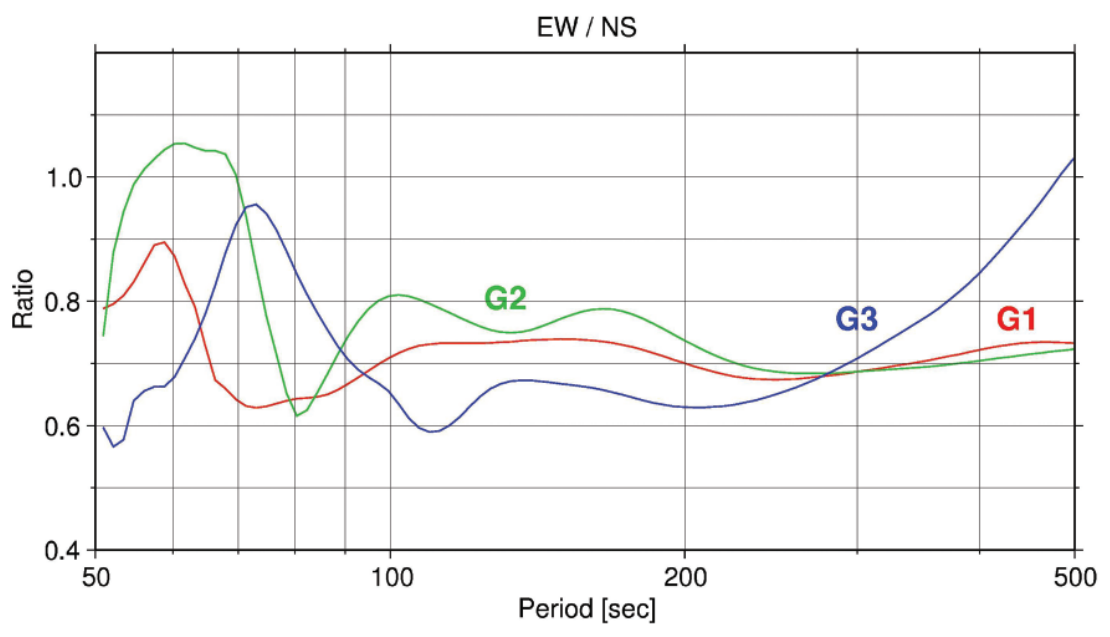

Fig. 8. Cross-sections of spectrograms in Fig. 7c calculated along the positions of local maximum amplitudes corresponding to G1(red), G2(green), and G3(blue) phases.

(a)

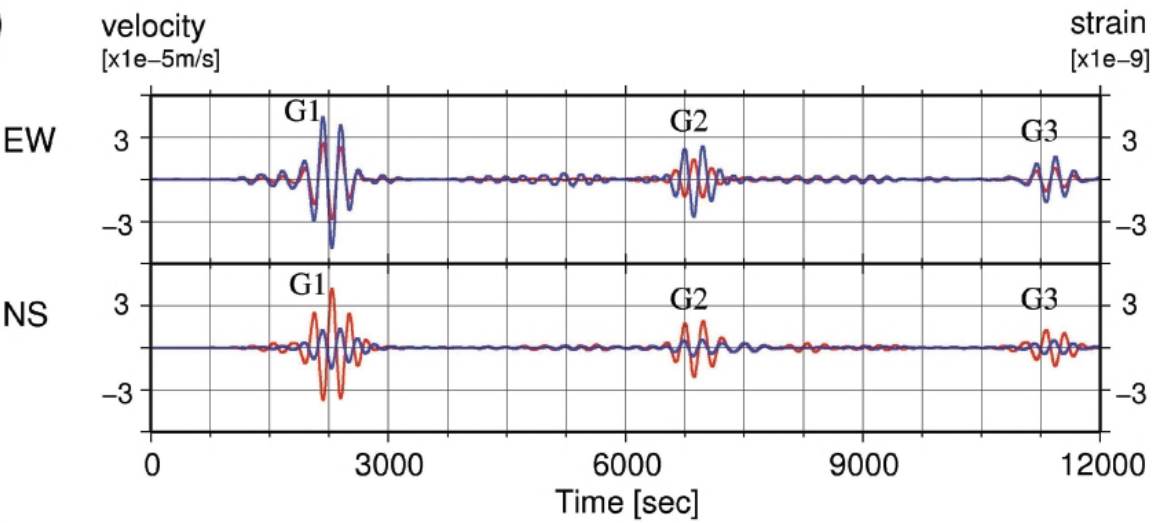

(b)

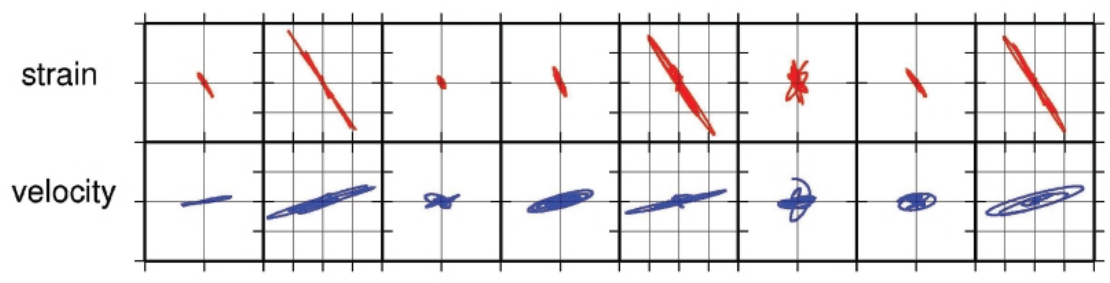

Fig. 9. (a) Observed strain (red) and velocity (blue) seismograms filtered in a period range of 171 - $400 \mathrm{sec}$. (b) Particle motions for the strain (red) and velocity (blue) seismograms. 


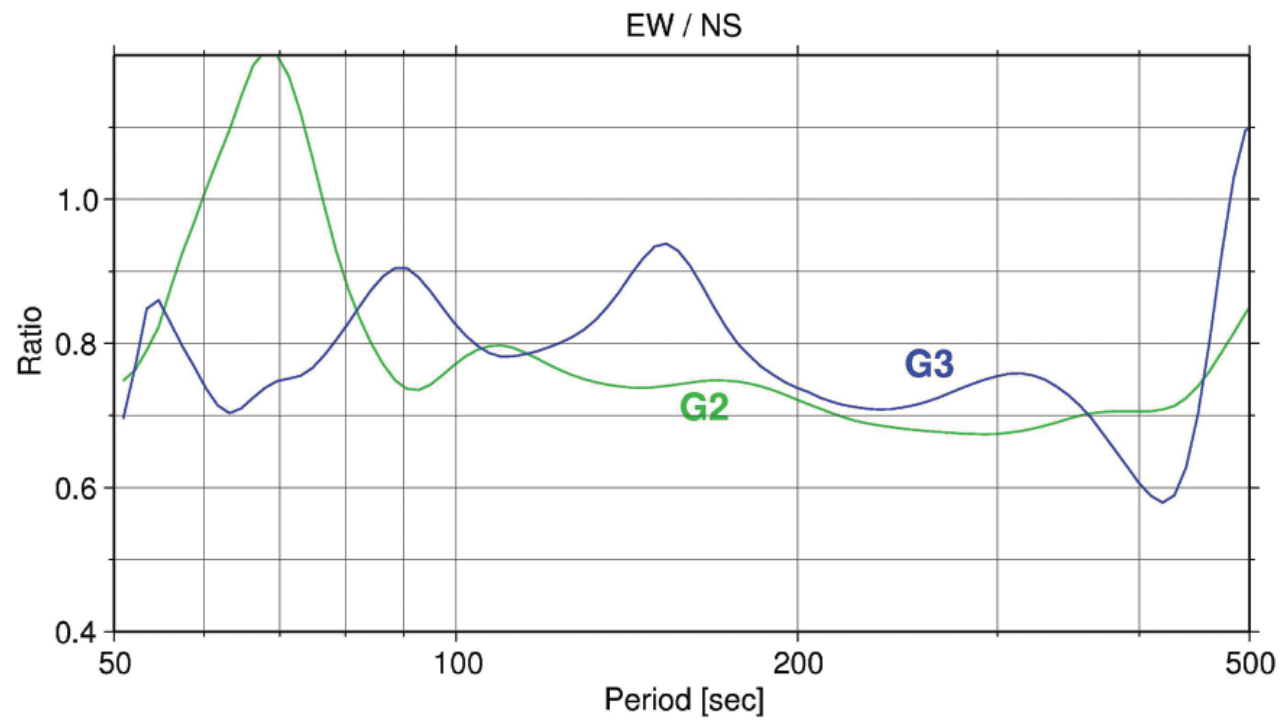

Fig. 10. Ratios of EW to NS component observed strain seismograms from Northern Chile Earthquake of 13 June 2005. The ratios are calculated for G2(green) and G3(blue) phases.

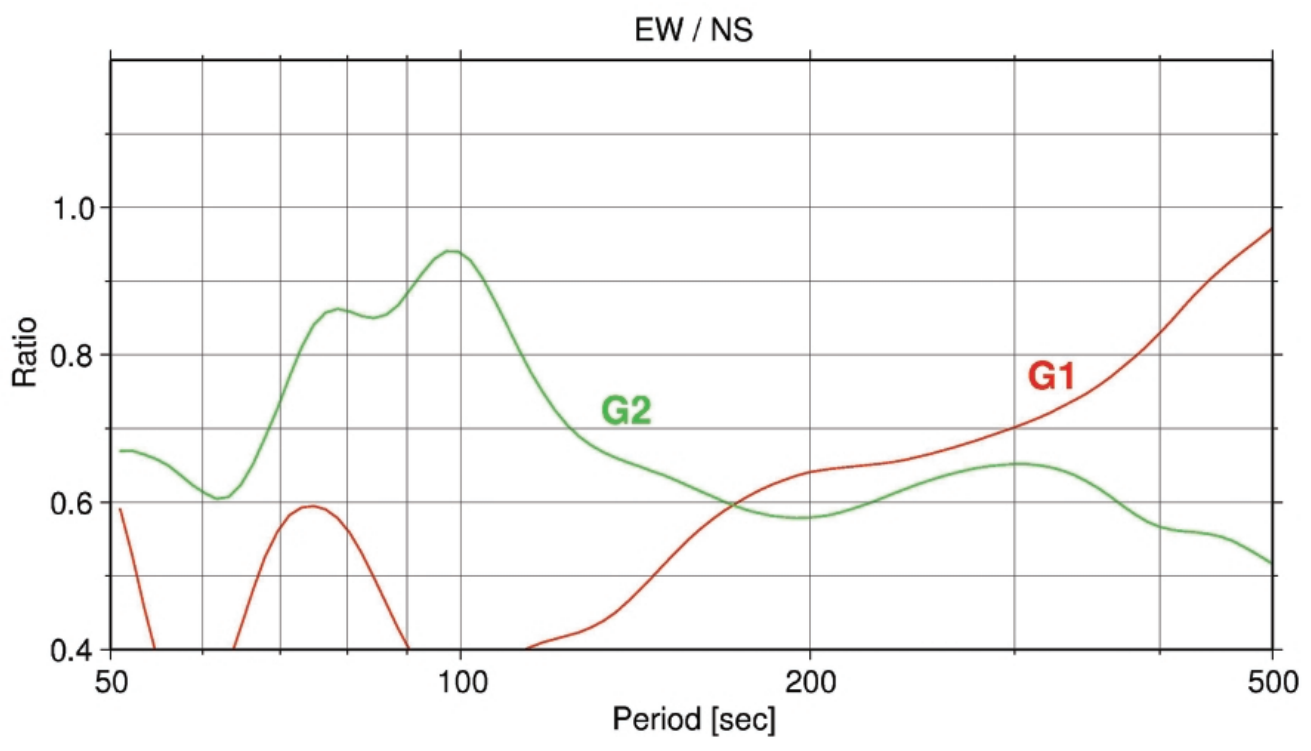

Fig. 11. Ratios of EW to NS component observed strain seismograms from Southern Peru Earthquake of 23 June 2001. The ratios are calculated for G1 (red) and G2 (green) phases. 
approximately 0.7 especially in the period range of $200-300 \mathrm{sec}$, although the results are less stable than that for 2004 Macquarie Island Earthquake because of contamination of Rayleigh waves.

From the above results, if NS component strain is corrected by being multiplied by 0.7 , EW and NS component strain can have the same amplitude for a Love wave. The amplitude of EW component strain, however, may have also been affected by local geology structure. In the following section, we compare the strain seismograms with velocity seismograms for evaluation of the effect.

\section{AMPLITUDE RATIO OF VELOCITY TO STRAIN SEISMOGRAM AND COM- PARISON WITH THEORETICAL PHASE VELOCITY}

At Matsushiro, seismic observation has also been performed by STS-1 broadband seismometers. Intercomparison of records from strain and velocity seismometers installed at a single station gives a local phase velocity of the wave (Mikumo and Aki 1964; Sacks et al. 1976). If both the records have not been affected by local geology, the phase velocities obtained by the method should be in agreement with theoretical values. To the contrary, if the comparison shows disagreement, at least one of them might have been affected (contraposition in logic). Thus, in this section, to investigate local effects on strain seismograms, we estimate local phase velocity from strain and velocity seismograms, and compare it with theoretical phase velocity for a Love wave. The local effects on velocity seismograms will be examined in the following section.

Consider a plane wave with an angular frequency $\omega$ travelling in a $\theta$-direction from the north. We take $x_{R}$-axis in the $\theta$-direction and make $x_{T}$-axis perpendicular to $x_{R}$-axis clockwise. $\omega$ is assumed very small and we consider only long-period surface waves in this study.

Then the displacement field can be expressed by:

$$
\left(\begin{array}{l}
u_{R}\left(\omega, t, x_{R}\right) \\
u_{T}\left(\omega, t, x_{R}\right)
\end{array}\right)=\left(\begin{array}{l}
A_{R}(\omega) \exp \left[i\left\{k_{R}\left(c_{R}(\omega) t-x_{R}\right)+\phi_{R}\right\}\right] \\
A_{T}(\omega) \exp \left[i\left\{k_{L}\left(c_{L}(\omega) t-x_{R}\right)+\phi_{L}\right\}\right]
\end{array}\right),
$$

where $u_{R}$ and $u_{T}$ denote radial and transverse component displacement, $A_{R}$ and $A_{L}$ amplitude of Rayleigh and Love waves, $k_{R}$ and $k_{L}$ wavenumber of Rayleigh and Love waves along the $x_{R}$-axis, $c_{R}$ and $c_{L}$ phase velocity for Rayleigh and Love waves, and $\phi_{R}$ and $\phi_{L}$ initial phase of Rayleigh and Love waves at $x_{R}$, respectively.

The phase velocities can be calculated by:

$$
\left(\begin{array}{c}
c_{R}(\omega) \\
c_{L}(\omega)
\end{array}\right)=\left(\begin{array}{l}
\left|\frac{\partial u_{R}}{\partial t}\right| /\left|\frac{\partial u_{R}}{\partial x_{R}}\right| \\
\left|\frac{\partial u_{T}}{\partial t}\right| /\left|\frac{\partial u_{T}}{\partial x_{R}}\right|
\end{array}\right) .
$$


Among the four derivatives, $\partial u_{R} / \partial t$ and $\partial u_{T} / \partial t$ can be obtained from horizontal component velocity seismograms by simple rotation. To obtain $\partial u_{R} / \partial x_{R}$ and $\partial u_{T} / \partial x_{R}$ from horizontal component strain seismograms, we utilize the following relation:

$$
\left(\begin{array}{c}
\varepsilon_{E E} \\
\varepsilon_{N N}
\end{array}\right)=\left(\begin{array}{cc}
\sin \theta \cos \theta & \sin ^{2} \theta \\
-\sin \theta \cos \theta & \cos ^{2} \theta
\end{array}\right)\left(\begin{array}{l}
\partial u_{T} / \partial x_{R} \\
\partial u_{R} / \partial x_{R}
\end{array}\right),
$$

or

$$
\left(\begin{array}{l}
\partial u_{T} / \partial x_{R} \\
\partial u_{R} / \partial x_{R}
\end{array}\right)=\left(\begin{array}{cc}
1 / \tan \theta & -\tan \theta \\
1 & 1
\end{array}\right)\left(\begin{array}{l}
\varepsilon_{E E} \\
\varepsilon_{N N}
\end{array}\right),
$$

where $\varepsilon_{E E}$ and $\varepsilon_{N N}$ denote linear strain in the direction of EW and NS, respectively. Note that shear strain contribution can be disregarded because we now deal with only long-period waves (Okubo et al. 2005).

Mikumo and Aki (1964) determined phase velocities by taking ratio of velocity and strain seismograms using no filter. However, it should be noted that surface waves with different frequencies have different phase velocities and that both the seismograms used here mainly consist of dispersed surface waves, in the period range of interest. Thus we extend their method as follows: We first prepare spectrograms by applying the multiple filter technique (Dziewonski et al. 1969) to both strain and velocity seismograms and then take their ratio on the timefrequency plane to estimate the phase velocity. This method allows us to obtain phase velocities of various seismic phases having various frequencies.

We first apply this method to theoretical seismograms to confirm its applicability as in the previous section. Figures $12 \mathrm{a}-\mathrm{c}$ show spectrograms for $\partial u_{T} / \partial t$ calculated from theoretical velocity seismogram, $\partial u_{T} / \partial x_{R}$ from theoretical strain, and their amplitude ratio which should correspond to local phase velocity of the Love wave. Figure 13 plots cross-sections of Fig. 12c along the positions of theoretical dispersion curves for G1, G2, and G3 phases. It is clear that the estimated phase velocity from the theoretical seismograms is consistent with the theoretical value, as we expected, in the period range of 100 - $500 \mathrm{sec}$.

Observed strain and velocity seismograms are analysed in the same way. Figures $14 \mathrm{a}-\mathrm{c}$ show spectrograms for $\partial u_{T} / \partial t$ calculated from observed velocity seismogram, $\partial u_{T} / \partial x_{R}$ from observed strain, and their amplitude ratio, respectively. The velocity seismograms have been corrected for instrumental response, and the strain seismograms have also been corrected because a first order high-pass filter with a cutoff period of 1000-sec has been applied to the both component strain seismograms before A/D conversion. In addition to that, NS component strain has been multiplied by 0.7 because of the result in the previous section.

Figure 15 plots cross-sections of Fig. 14c along the positions of local maximum amplitudes corresponding to G1, G2, and G3 phases and shows that the estimated phase velocity from the observed seismograms is approximately $24 \%$ smaller than the theoretical value in the period range of $120-500 \mathrm{sec}$, unlike the result for the theoretical seismogram. It seems that the above factor (24\%) does not depend on the period within this period range again. 

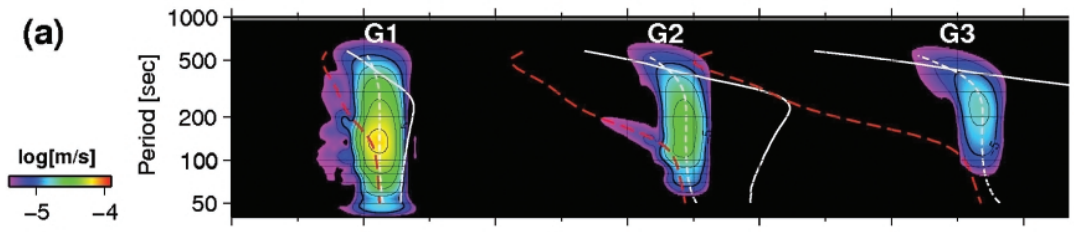

(b)
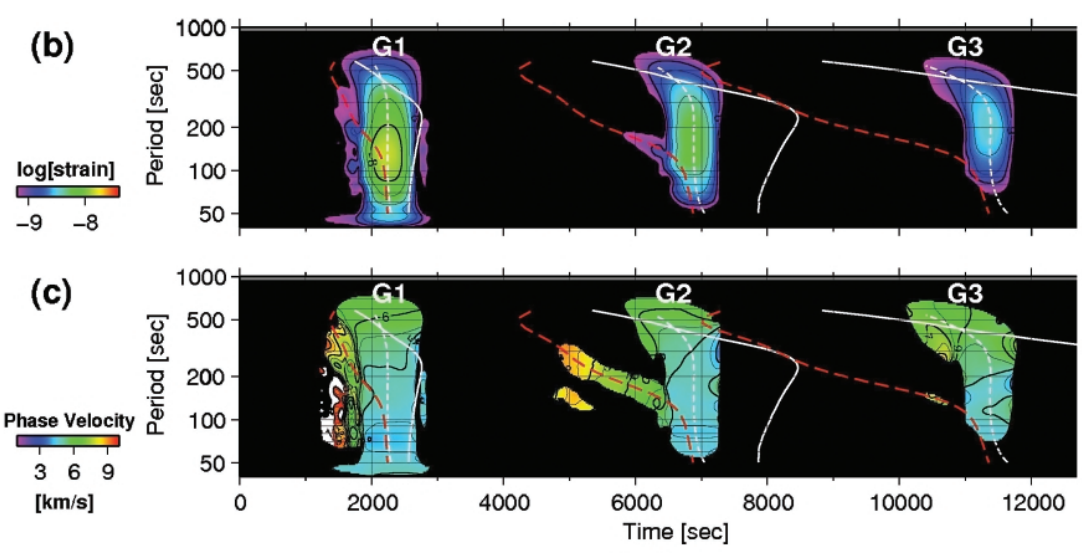

Fig. 12. Spectrograms for (a) $\partial u_{T} / \partial t$ and (b) $\partial u_{T} / \partial x_{R}$ calculated from theoretical velocity and strain seismograms at Matsushiro for the 2004 North of Macquarie Island earthquake, and (c) the ratio of $\partial u_{T} / \partial t$ to $\partial u_{T} / \partial x_{R}$, corresponding to Love wave phase velocity. The time and curves plotted are the same as in Fig. 4.

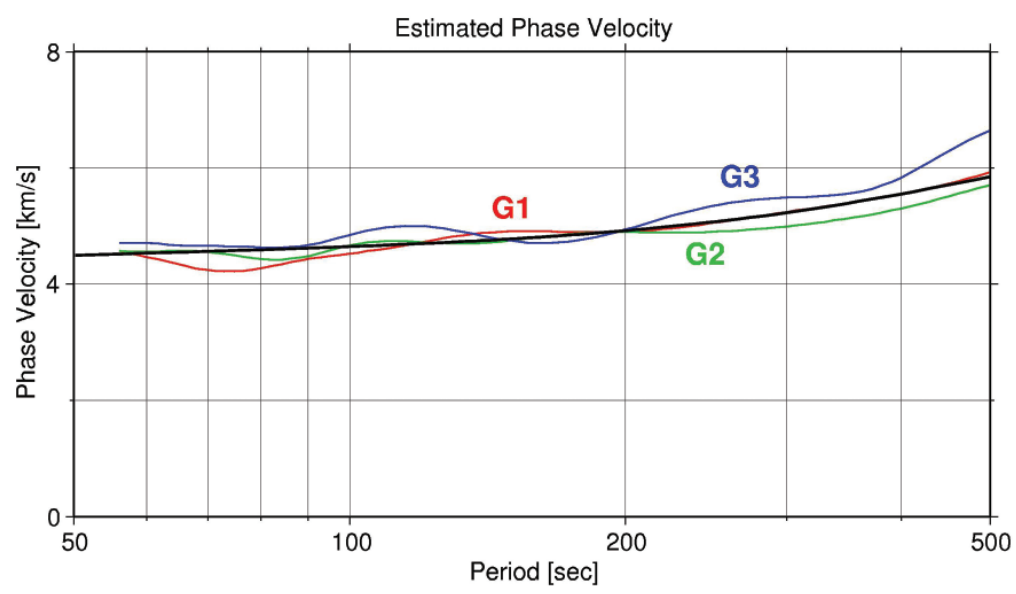

Fig. 13. Cross-sections of Fig. 12c calculated along the positions of theoretical dispersion curves for G1 (red), G2 (green), and G3 (blue) phases. The black curve is theoretical phase velocity for fundamental mode Love wave in PREM. 

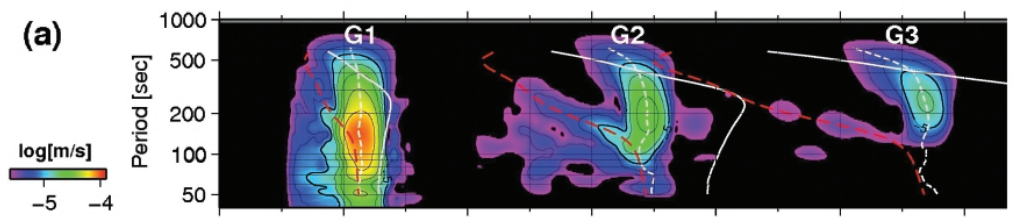

(b)
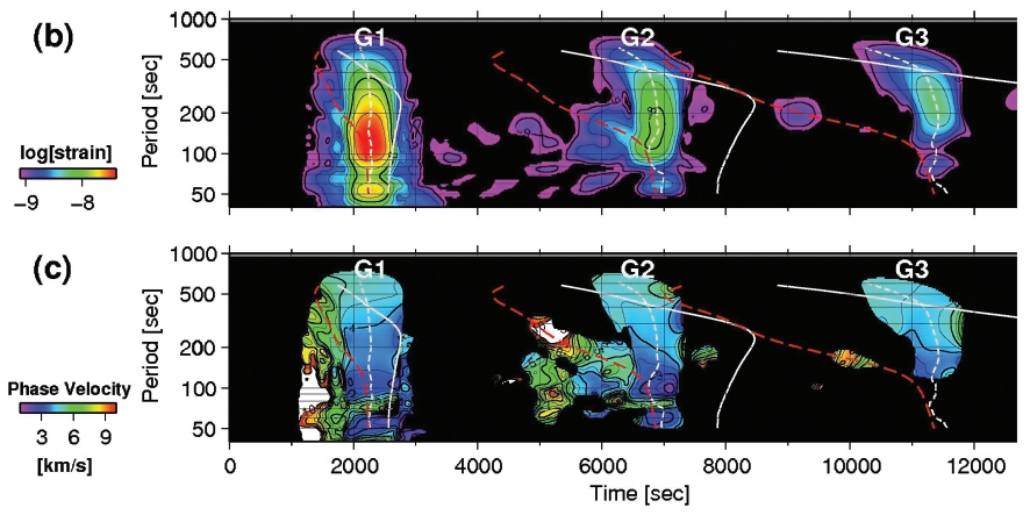

Fig. 14. Spectrograms for (a) $\partial u_{T} / \partial t$ and (b) $\partial u_{T} / \partial x_{R}$ calculated from observed velocity and strain seismograms at Matsushiro from the 2004 North of Macquarie Island earthquake, and (c) the ratio of $\partial u_{T} / \partial t$ to $\partial u_{T} / \partial x_{R}$, corresponding to Love wave phase velocity. The time and curves except for the white dotted curves are the same as in Fig. 4. The white dotted curves denote the position of local maximum amplitudes corresponding to G1, G2, and G3 phases.

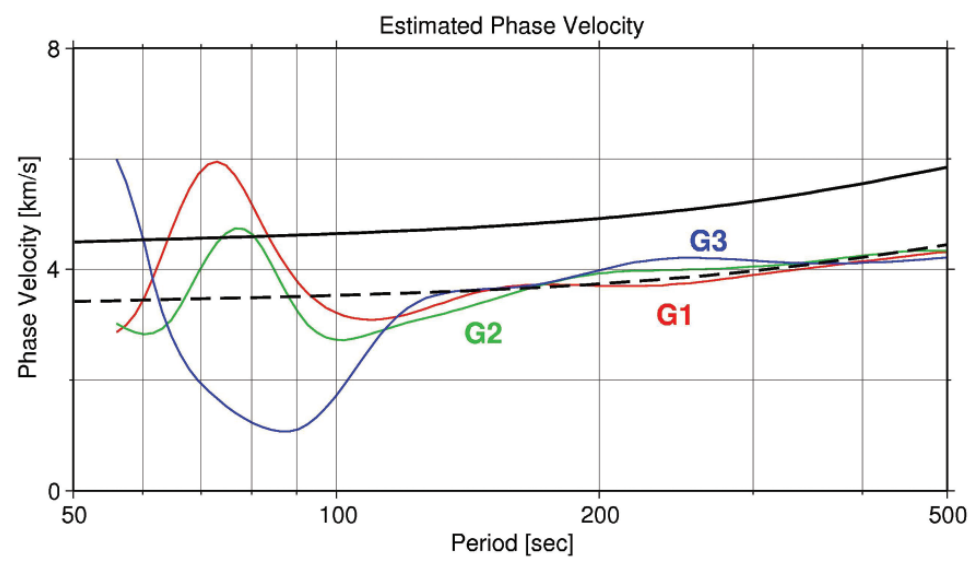

Fig. 15. Cross-sections of Fig. 14c calculated along the positions of local maximum amplitudes corresponding to G1 (red), G2 (green), and G3 (blue) phases. The solid black curve is the same as in Fig. 13. The dotted black curve is theoretical phase velocity multiplied by 0.76 . 
It follows from this result that the EW and NS component strain seismograms for a Love wave have been amplified by factors of $1.32(=1 / 0.76)$ and $1.88(=1.32 / 0.7)$, respectively, if velocity seismograms have not been affected by local geology structure. The local effects on velocity seismograms will be examined in the following section.

\section{COMPARISON OF VELOCITY SEISMOGRAM WITH OTHER SEISMOGRAMS AT F-NET STATIONS}

In this section, we compare the velocity seismogram observed by STS-1 seismometer at Matsushiro with other velocity seismograms observed by STS-1 seismometers at F-net stations near Matsushiro and by STS-2 seismometer at Matsushiro to investigate the local effect on the observed velocity seismogram.

Figure 16 is a map of observation stations used. All stations have nearly the same epicentral distance and azimuth from epicenter as Matsushiro (every deviation from Matsushiro is less than $2 \%$ ), and differences in radiation pattern and distance attenuation are very small.

In Figs. 17 and 18 we show the comparison of velocity spectra, which are cross-sections of spectrograms for transverse component theoretical (Fig. 17) and observed (Fig. 18) velocity seismograms at Matsushiro and F-net stations, along positions of local maximum amplitudes

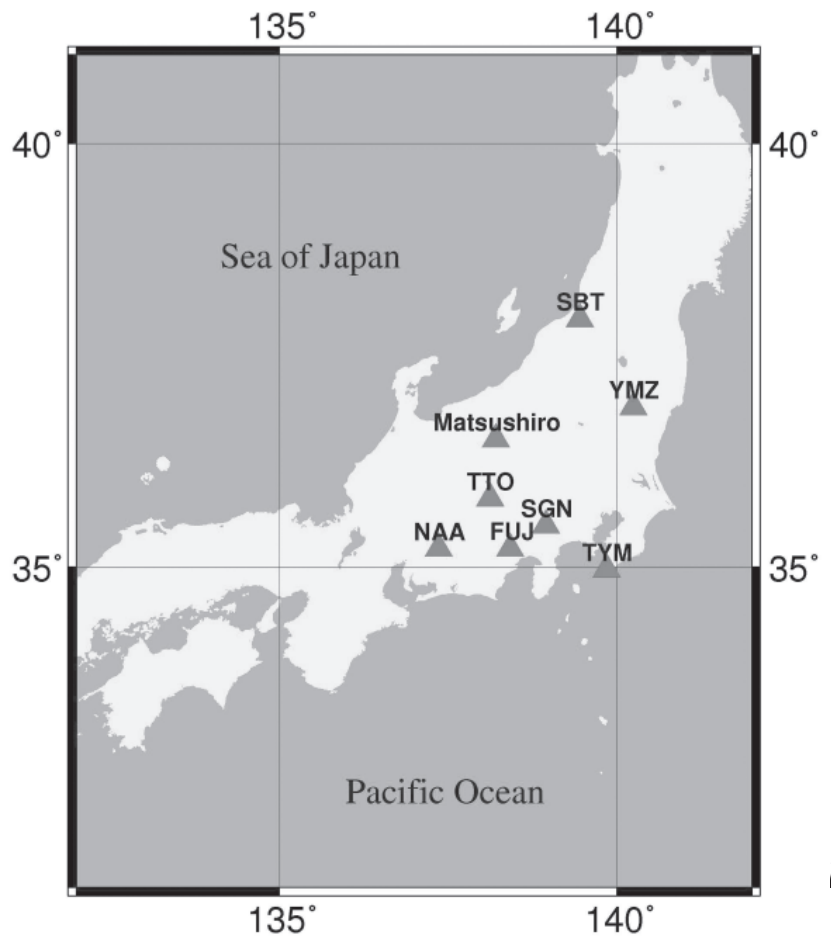

Fig. 16. Map of observation stations. 


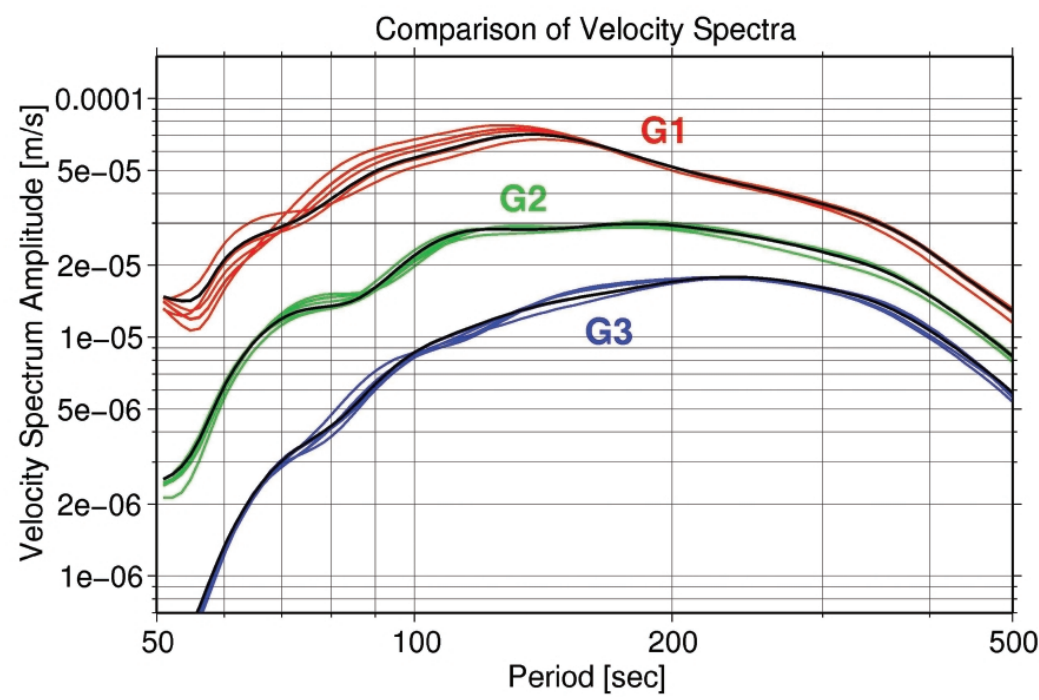

Fig. 17. Cross-sections of spectrograms for transverse component theoretical velocity seismograms at Matsushiro (black) and F-net stations (coloured) along the positions of theoretical dispersion curves for G1, G2, and G3 phases.

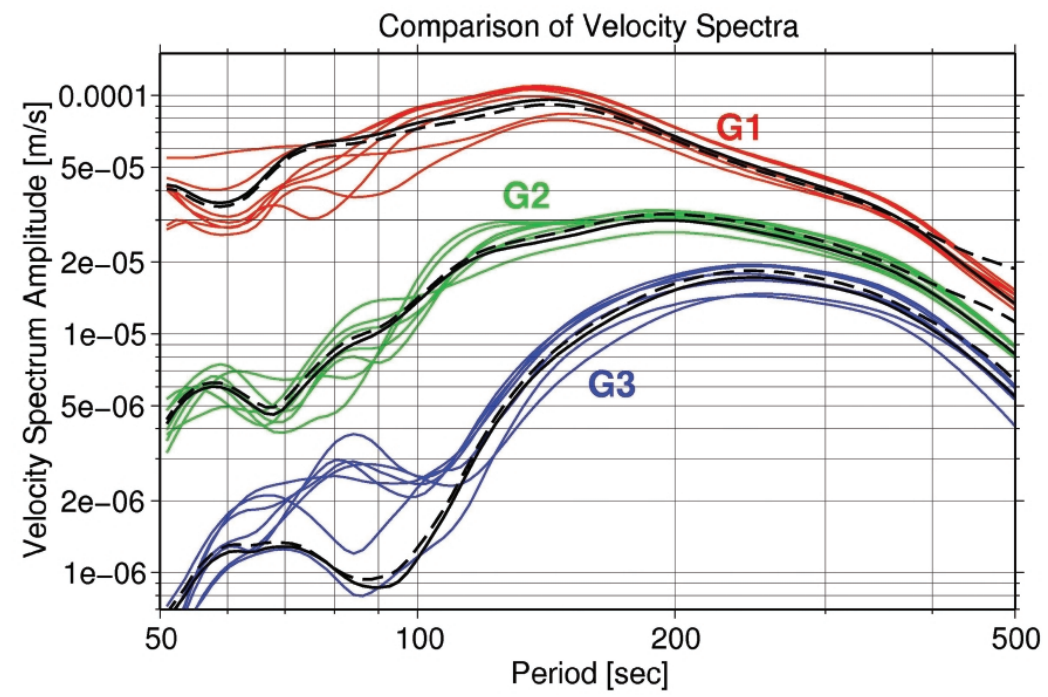

Fig. 18. Cross-sections of spectrograms for transverse component observed velocity seismograms at Matsushiro (black) and F-net stations (coloured) along the positions of local maximum amplitudes corresponding to G1, G2, and G3 phases. The Solid black curves and the dotted black curves are for seismograms recorded by STS-1 and STS-2, respectively. 
corresponding to G1, G2, and G3 phases. In Fig. 17, all spectra have nearly the same amplitudes in the period range of $150-500 \mathrm{sec}$. In Fig. 18, the velocity spectra observed at different stations have slightly different amplitudes, but their deviations are less than $10 \%$. Thus we consider the velocity seismograms observed at all observation stations including Matsushiro have been nearly unaffected.

\section{DISCUSSION AND CONCLUSION}

We proposed a simple method to evaluate local effects on a strain seismogram and applied the method to investigation of the effects at Matsushiro for a Love wave in a period range of 170 - $400 \mathrm{sec}$. The results are summarized as follows:

1. Amplitude ratio of EW to NS component is $30 \%$ smaller than theoretical value.

2. Amplitude ratio of velocity to strain is $24 \%$ smaller than theoretical value.

3. Above two factors are independent of period.

4. Velocity seismograms are nearly unaffected by local structure.

From these results, we conclude that observed EW and NS component linear strain seismograms have been amplified by factors of 1.32 and 1.88, respectively, and must be divided by the factors to remove the local effects.

In this section, we finally compare observed strain seismograms before and after the correction with theoretical strain seismograms to check the accuracy of the factors. Figures 19 and 20 show the theoretical and observed strain seismograms. All seismograms have been filtered in the period range of $171-400 \mathrm{sec}$. In Fig. 20, the observed strain seismograms have been divided by above factors, unlike in the case of Fig. 19. In Fig. 20, the observed and theoretical seismograms are in good agreement, whereas in Fig. 19, the observed seismograms have remarkably larger amplitudes than theoretical strains. Note that the above factors are not what were determined to match these waveforms. This comparison also indicates that local effects must be removed from observed strain seismograms before we use the observed strain seismogram for seismological analyses, and that above factors are practical enough for the correction.

Although it is not so widely recognized, some researchers have already pointed out the fact that small-scale heterogeneity may cause large change in strain amplitude even in a longperiod range as follows:

1. Taniguchi and Oike (1984) investigated distribution of strain change in a single observation vault associated with Earth tides and with arrival of teleseismic surface waves with periods less than 1-min, and found that the strain amplitudes at a fracture zone were approximately twice as large as those at neighbouring sections both for the Earth tides and for the surface waves.

2. At Matsushiro, observation of crustal movement was performed by a 25-meter-long laser extensometer installed in a north-south direction and about 150 meters apart from the NS 
(a)

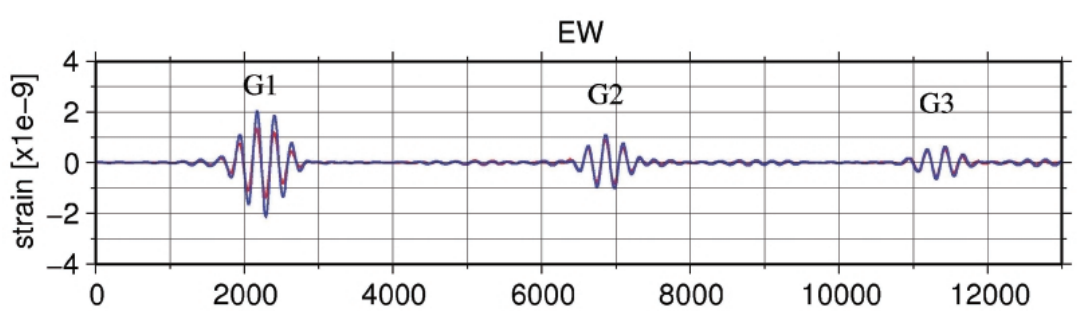

(b)

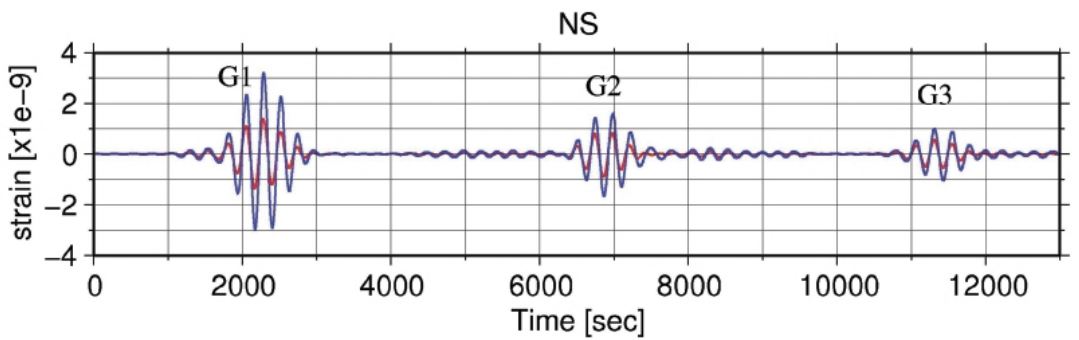

Fig. 19. Theoretical (red) and observed (blue) strain seismograms. (a) EW and (b) NS component. All seismograms have been filtered in the period range of $171-400 \mathrm{sec}$.

(a)

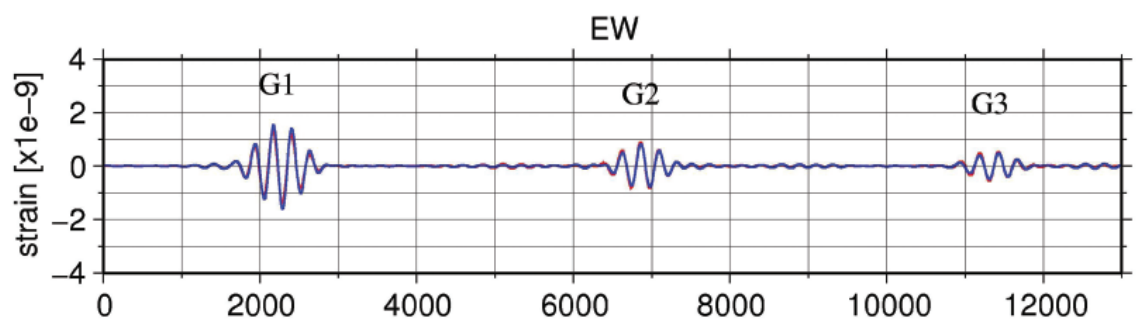

(b)

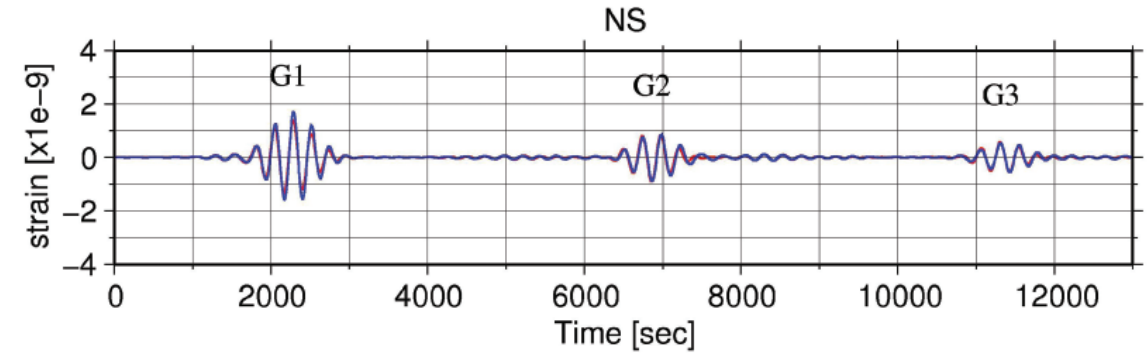

Fig. 20. Theoretical (red) and observed (blue) strain seismograms. (a) EW and (b) NS component. All seismograms have been filtered in the period range of $171-400 \mathrm{sec}$. The observed strain seismograms have been divided by 1.32 for EW component and 1.88 for NS. 
component quartz-tube extensometer about 30 years ago (Ohishi et al. 1976). Hamada and Kashiwabara (1981) found that the observed strain amplitudes associated with Earth tides by the laser extensometer were approximately $30 \%$ smaller than those by the NS component quartz-tube extensometer.

Thus the results obtained in the present study are not surprising, although the local effects in the period range of 170 - $400 \mathrm{sec}$ have never been investigated. For a Rayleigh wave, however, we have found that local effects on a strain seismogram at Matsushiro are quite different from those for a Love wave. The results will be reported in the near future (Okamoto et al. 2007).

Acknowledgements The authors appreciate Dr. Ruey-Der Hwang and anonymous reviewer's comments which have considerably improved the quality of our paper. The broadband velocity seismograms used in this study were provided by the Incorporated Research Institutions for Seismology and the National Research Institute for Earth Science and Disaster Prevention. The fortran source code to synthesize theoretical seismograms was provided by Dr. Osamu Kamigaichi, Japan Meteorological Agency. Figures have been prepared with the GMT package provided by Wessel and Smith (1995).

\section{REFERENCES}

Asai, Y., M. Okubo, H. Ishii, H. Aoki, T. Yamauchi, Y. Kitagawa, N. Koizumi, and S. Azuma, 2005: Co-seismic strain-steps associated with the 2004 off the Kii peninsula earthquakes - Observed with Ishii-type borehole strainmeters and quartz-tube extensometers. Earth Planets Space, 57, 309-314.

Benioff, H., and B. Gutenberg, 1952: The response of strain and pendulum seismographs to surface waves. Bull. Seismol. Soc. Am., 42, 229-237.

Dziewonski, A., S. Bloch, and M. Landisman, 1969: A technique for the analysis of transient seismic signals. Bull. Seismol. Soc. Am., 59, 427-444.

Dziewonski, A. M., and D. L. Anderson, 1981: Preliminary reference earth model. Phys. Earth Planet. Inter., 25, 297-356.

Gilbert, F., and A. M. Dziewonski, 1975: An application of normal mode theory to the retrieval of structural parameters and source mechanisms from seismic spectra. Phil. Trans. Roy. Soc. London., A278, 187-269.

Hamada, N., and S. Kashiwabara, 1981: Some problems related to crustal movement observations at Matsushiro. Quart. J. Seismol., 45, 49-83. (in Japanese)

Herrmann, R., 1969: Some aspects of band-pass filtering of surface waves. Bull. Seismol. Soc. Am., 63, 663-671.

Ikegami, Y., 2006: Synthesis of theoretical strain seismograms for Sumatra-Andaman Earthquake of December 26, 2004 and comparison with observed waveforms. Master Thesis, Kyoto University, Kyoto, Japan. (in Japanese)

Mikumo, T., and K. Aki, 1964: Determination of local phase velocity by intercomparison of seismograms from strain and pendulum instruments. J. Geophys. Res., 69, 721-731. 
Ohishi, T., S. Seino, and Y. Sakurai, 1976: Laser extensometer for Earth strain measurement. J. Geod. Soc. Japan, 22, 288-289.

Okamoto, T., Y. Ikegami, and K. Kokubo, 2007: Local Effects on Strain Seismograms at Matsushiro Seismological Observatory - 2. Rayleigh waves. Terr. Atmos. Ocean. Sci., accepted.

Okubo, M., Y. Asai, H. Aoki, and H. Ishii, 2005: The seismological and geodetical roles of strain seismogram suggested from the 2004 off the Kii peninsula earthquakes. Earth Planets Space, 57, 303-308.

Sacks, I. S., J. A. Snoke, R. E. G. King, and J. Beavan, 1976: Single site phase velocity measurement. Geophys. J. R. astr. Soc., 46, 253-258.

Taniguchi, K., and K. Oike, 1984: Behavior of fractured zones at the Yamasaki Fault for teleseismic surface waves. J. Phys. Earth, 32, 449-461.

Wakui, S., Y. Nishimae, T. Tokumoto, T. Furudate, and H. Tatehata, 2002: Crustal Movement Data Processing with Renewal of the Strain Seismograph Telemeter System. Technical Reports of the Matsushiro Seismological Observatory, JMA, 19, 143-150. (in Japanese)

Wessel, P., and W. H. F. Smith, 1995: A new version of the Generic Mapping Tools (GMT). EOS Trans. AGU, 76, $329 \mathrm{pp}$.

Yamagishi, Y., S. Izumi, and K. Aihara, 1976: Observation of Crustal Deformation at Matsushiro. Quart. J. Seismol., 41, 13-20. (in Japanese)

Okamoto, T., Y. Ikegami, and K. Kokubo, 2007: Local effects on strain seismogram at Matsushiro seismological observatory - 1. love waves. Terr. Atmos. Ocean. Sci., 18, 547-565, doi: 10.3319/TAO.2007.18.3.547(T). 